\title{
State Estimation Based Neural Network in Wind Speed Forecasting: A Non Iterative Approach
}

\author{
D. Rakesh Chandra ${ }^{1, *}$, M. Sailaja Kumari ${ }^{2}$, \\ M. Sydulu ${ }^{2}$ and V. Ramaiah ${ }^{1}$ \\ ${ }^{1}$ Department of Electrical Engineering, KITS, Warangal, \\ Telangana-506015, India \\ ${ }^{2}$ Department of Electrical Engineering, National Institute of Technology Warangal, \\ Telangana-506004, India \\ E-mail: rksh.chndr@gmail.com \\ * Corresponding Author
}

Received 08 February 2018; Accepted 01 September 2018;

Publication 24 September 2018

\begin{abstract}
Renewable energy sources have gained a lot of importance in today's power generation. These sources of energy are pollution free and freely available in nature. Wind is the most prominent energy source among the renewable energy sources. Increased wind penetration into the existing power system will create reliability problems for grid operation and management. Wind speed forecasting is an important issue in wind power grid integration as it is chaotic in nature. This paper presents a new State Estimation based Neural Network (SENN) for day ahead (24 hours ahead) wind speed forecasting and its performance has been compared with traditional Back Propagation Neural Network (BPNN). SENN is a non iterative technique, where the weights between input-hidden and hidden-output layers are estimated using a Weighted Least Square State Estimation (WLSSE) approach. It accounts noise associated with input and output data, giving accurate results without any iteration. This method is quite efficient and faster compared to conventional Back Propagation Neural Network (BPNN).
\end{abstract}

Journal of Green Engineering, Vol. 8_3, 263-282. River Publishers

doi: 10.13052/jge1904-4720.833

This is an Open Access publication. (c) 2018 the Author(s). All rights reserved. 
Keywords: Back Propagation Neural Network, State Estimation Neural Network, Wind forecast and Weighted Least Square.

\begin{tabular}{ll}
\hline Abbreviations: \\
\hline BPNN & Back Propagation Neural Network \\
SENN & State Estimation Neural Network \\
WLSSE & Weighted Least Square State Estimation \\
BPA & Back Propagation Algorithm \\
SVM & Support Vector Machine \\
ANN & Artificial Neural Network \\
\hline
\end{tabular}

\section{Introduction}

The significance of renewable energy sources has recently increased due to multiple factors, including their main property of being eco-friendly. Global warming and limited availability of fossil fuels are also leading to making renewable energy sources the best alternative energy sources. Among the renewable energy sources, wind power is most promising and rapidly expanding worldwide. With the introduction of deregulation in electricity market many new challenges have been taking place by the participants of power industry. Thus, wind speed forecasting is becoming a major issue in deregulated market as wind power is an important contribution to the power industry. Wind speed forecasting reflects directly on power forecasting as wind power is proportional to the cube of wind speed. An accurate wind power forecasting can mitigate undesirable consequences in wind connected power systems and it is also helpful in power system planning studies. As on date, many forecasting methods exist in literature.

A novel hybrid intelligent algorithm has been introduced in [1] for wind power forecasting which uses fuzzy network and wavelet transform, and it is optimized by firefly optimization algorithm. Khosravi et al. [2] Investigated short term forecasting of wind power using two neural network based methods for rapid and direct construction of prediction intervals. A statistical method has also been proposed in [3] for power forecasting where wavelet decomposition is used to predict wind power up to 30 hours. Wavelets are also used for wind speed forecasting in [4-6] and different wavelet functions with various decomposition levels were used to estimate the wind speed. They are also used for energy price forecasting [7]. Sideratos et al. [8] have used a new learning method for radial basis function neural network and 
this has been tested on a real wind farm and then compared with state-ofart models. Various wind speed forecasting methods and their significance in the present power system scenario has been discussed in $[9,10]$. A dayahead wind speed forecasting along with direction has been investigated in [11] and results are compared with persistent models. The wind power forecasting is the prerequisite for integrating wind power into the grid as it links the available wind power to the conventional power [12]. In [13] four time series models were examined with different prediction horizons built by data mining algorithm. Barbounis et al. [14] deals with long term wind speed and power forecasting using local recurrent neural networks based on meteorological information. Artificial neural networks have also been used for short-term wind power forecasting [15]. Fuzzy logic and neural network based wind speed forecasting method has been proposed in [16] this method is fast in computation and quite accurate in wind speed prediction. A hybrid forecasting method which is a combination of autoregressive integrated moving average, Artificial Neural Network (ANN), Support Vector Machine (SVM) has been used in [17] for wind speed and power forecasting for different time horizons. A combined approach consisting of ANN, SVM, fuzzy inference system has been used again for wind speed forecasting to improve forecasting accuracy [18]. ANFIS and fuzzy logic techniques have been used extensively for load forecasting [19]. A specific commercial software package has been developed to study wind power forecast in [20] using historical data for this purpose. Finally the wind forecasting also shows its impact on power system reliability and this topic has been extensively discussed in [21] and [22].

In this paper, a State Estimation based Neural Network (SENN) is proposed as a wind forecasting tool and tested on one year real wind data in the Midwest ISO region. SENN based wind speed forecasting method is a novel approach, as it is non iterative. The weights are estimated more accurately with the mathematical approach (which will be explained in the coming sections) at the first instant itself there by forecasting results are more reliable. Moreover, this method is compared with another well-assessed wind speed forecasting method, namely Back-Propagation Neural Network (BPNN). This paper is organized as follows: Section 2 presents traditional BPNN architecture, Section 3 describes the proposed SENN based wind speed forecasting method, Section 4 analyses the results of the two respective methods and Section 5 concludes the paper. 


\section{BPNN Architecture}

In this section application of ANN wind speed forecasting is explained. ANN can utilize past history of data to train the network and forecast future data. ANN based methods are suitable for forecasting compared to traditional methods as they are adaptable in nature. Here, Back Propagation Algorithm (BPA) is used for wind speed forecasting. BPA is a supervised learning technique where the target (output) is known. The main mechanism behind BPA usage consists of back propagating the error and adjusting the weights in proportion to error gradient and this process repeats until error is minimized to the desired level. The considered ANN consists of 3 layers namely the input layer, the hidden layer, and the output layer as shown in Figure 1. Sigmoid function has been used as the activation function in the hidden layer and output layer. Generally a sigmoid function can handle non linear problems effectively and its mathematical expression is given by:

$$
\phi(x)=\frac{1}{1+e^{-x}}
$$

The proposed neural network uses 30 inputs, 45 hidden neurons and 1 output node. $\mathrm{X}_{1}, \ldots, \mathrm{X}_{30}$ are the input samples, IH points from input to hidden and $\mathrm{HO}$ points from hidden to output. $\mathrm{V}_{\mathrm{ij}}$ and $\mathrm{W}_{\mathrm{ij}}$ represent weights between input to hidden and hidden to output respectively. For training, 1 to 30 data samples are the input and $31^{\text {st }}$ is the output similarly $32^{\text {nd }}$ to $61^{\text {st }}$ data samples are the input and $62^{\text {nd }}$ is the output. This process is continued for 80 patterns, where

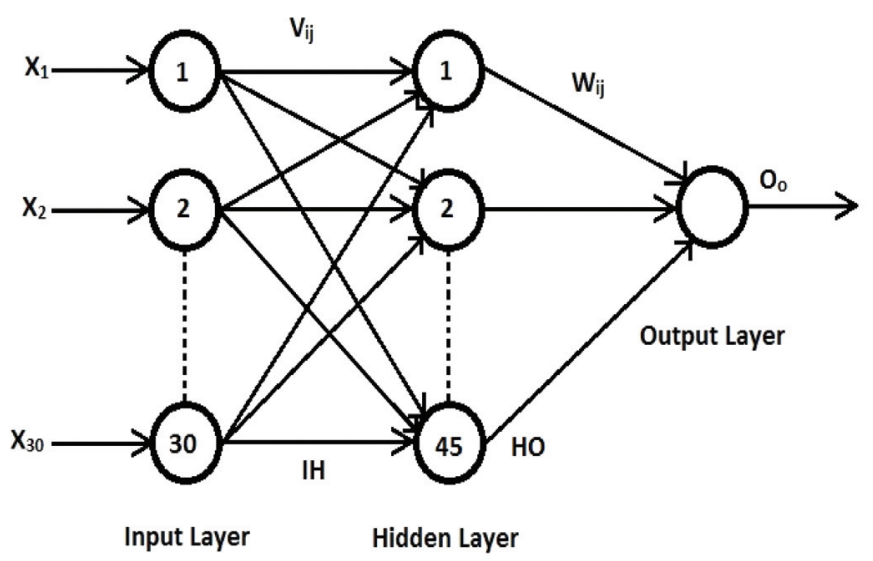

Figure 1 Neural network architecture used for both BPNN, SENN. 


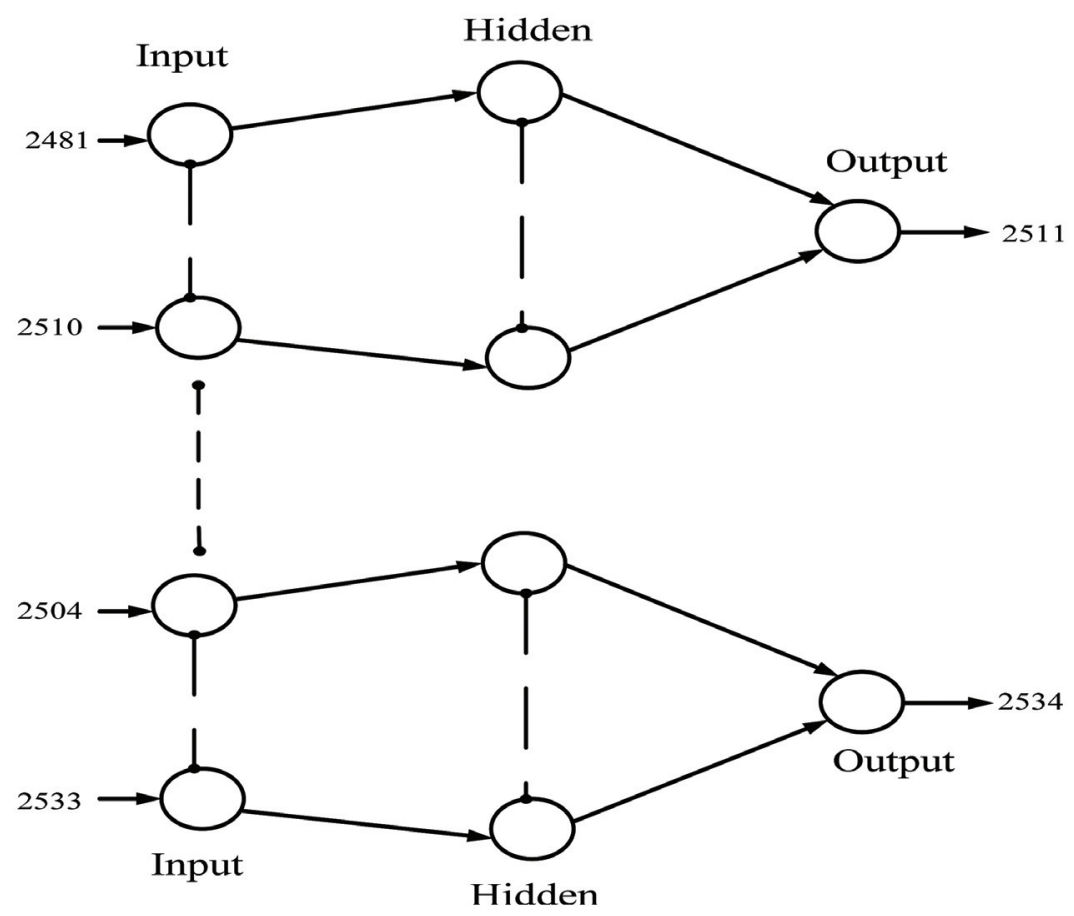

Figure 2 Conceptual graphic representation of neural network Testing phase.

the $80^{\text {th }}$ training pattern is 2450 to 2479 samples as input and 2480 as the output.

Once the problem converges, the converged weights are used for testing. For testing, sample numbers from 2481 to 2510 have been used as the input and the next 24 samples ( 2511 to 2534 ) are the forecasted outputs. At each instant, the forecasted output has been taken as the input for the next pattern. This recursive procedure is then used for the next 24 hours. Figure 2 explains the testing phase of the ANN used. A similar process has been adopted in case of SENN and it is discussed in the Section 3.

\section{State Estimation Based Neural Network (SENN)}

A State Estimation based Neural Network (SENN) is proposed and used for load forecasting in [23]. SENN is applied to wind speed forecasting in this paper for the first time. State estimation is the procedure of estimating 
the reliable state vector of the system from a given set of imperfect measurements made on the power system. A state estimator is able to nullify random errors in meter readings. Generally, this process involves deficient measurements that are redundant and the system state estimating process is involved with statistical approach that estimates actual values of the state variables to reduce the selected error criterion. A commonly used criterion is to reduce the sum of squares of the weighted differences between the "estimated" and "actual" (measured) value of a function as much as possible. Here a weighted least Square State Estimation (WLSSE) technique has been used to estimate the optimal weights between the layers of the Neural Network.

\subsection{Weighted Least Square (WLS) State Estimator}

In WLS estimation, the objective function is to minimize the sum of squares of the weighted deviations with respect to the true measurements as much as possible. WLS objective function is discussed as follows.

$$
\begin{gathered}
\underbrace{\min }_{\left(x_{1}, x_{2} \ldots x_{N S}\right)} J\left(x_{1}, x_{2} \ldots x_{N S}\right)=\sum_{i=1}^{N_{m}} \frac{\left[Z_{i}-f_{i}\left(x_{1}, x_{2} \ldots x_{N S}\right)\right]^{2}}{\sigma_{i}^{2}} \\
{[Z]=[f(x)]+[\eta]} \\
J(x)=[\eta]^{T} \cdot R^{-1} \cdot[\eta] \\
J(x)=[Z-[f(x)]]^{T} \cdot R^{-1} \cdot[Z-[f(x)]]
\end{gathered}
$$

Minimization of $J(x)$ would result in

$$
\begin{gathered}
x^{e s t}=\left[H^{T} R^{-1} H\right]^{-1} \times\left[H^{T} R^{-1}\right] Z^{\text {meas }} \\
{\left[x^{e s t}\right]=\left[H_{\text {effe }}\right]^{-1} \times\left[Z_{\text {effe }}\right]}
\end{gathered}
$$

Where

$$
\left[H_{\text {effe }}\right]=\left[H^{T} R^{-1} H\right] ;\left[Z_{e f f e}\right]=\left[H^{T} R^{-1} Z^{\text {meas }}\right]
$$

$J(x)$ - Objective function

$\mathrm{Z}$ - Actual measurements

$f(x)$ - True measurements

$[H]-\operatorname{An} N_{m}$ by $N_{s}$ matrix consisting of linear function coefficients $f_{i}(x)$.

$\sigma-$ Standard deviation

$\eta$ - Noise vector 
$N_{m}$ - Number of measurements

$N_{s}$ - Number of Training Samples

$\left[\mathrm{R}^{-1}\right]=$ Weight matrix (it is a diagonal matrix with $\frac{1}{\sigma_{i}{ }^{2}}$ as diagonal element)

In the proposed forecasting method, WLSSE technique is used between input layer to hidden layer, and hidden to output layers. In this technique, the weight matrices between the layers have been estimated like the states as $X_{1}, X_{2}, \ldots, X_{n}$. Thus to train the three layered neural network, a state estimation approach has been applied between input-hidden and hidden-output individually.

\subsection{SENN Based Wind Speed Forecasting}

SENN construction is similar to BPNN structure: except for the weight vector estimation, remaining actions and structure like normalization, denormalization, training period, node structure etc. remain unchanged. In SENN method weights need not be initialized randomly. As anticipated a sigmoid function has been used as activation function in the hidden layer. In this architecture again 30 input nodes, 45 hidden nodes and one output node are set, and the same network has been used for ANN based wind speed forecasting. A total number of 80 patterns are used and each pattern consists of 30 samples therefore a total of 2400 samples are $(80 * 30=2400)$ used for training.

\subsection{Mathematical Approach for SENN}

In this section the complete mathematical set of equations involved in SENN referring to Figure 1 and their formulation have been explained. Each individual training sample output is given by

$$
\begin{aligned}
& V_{11} \times x_{1}^{(1)}+V_{21} \times x_{2}^{(1)}+V_{31} \times x_{3}^{(1)}+\cdots+V_{301} \times x_{30}^{(1)}=y_{1}^{(1)} \\
& V_{11} \times x_{1}^{(2)}+V_{21} \times x_{2}^{(2)}+V_{31} \times x_{3}^{(2)}+\cdots+V_{301} \times x_{30}^{(2)}=y_{1}^{(2)} \\
& : \\
& \vdots \\
& V_{11} \times x_{1}^{(80)}+V_{21} \times x_{2}^{(80)}+V_{31} \times x_{3}^{(80)}+\cdots+V_{301} \times x_{30}^{(80)}=y_{1}^{(80)}
\end{aligned}
$$


The matrix form referred to above equations is given as below:

$$
\left[\begin{array}{cccc}
x_{1}^{(1)} & x_{2}^{(1)} & \cdots & x_{30}^{(1)} \\
x_{1}^{(2)} & x_{2}^{(2)} & \cdots & x_{30}^{(2)} \\
\vdots & \vdots & \cdots & \vdots \\
x_{1}^{(80)} & x_{2}^{(80)} & \cdots & x_{30}^{(80)}
\end{array}\right]_{(80 \times 30)} \times\left[\begin{array}{c}
V_{11} \\
V_{21} \\
\vdots \\
V_{301}
\end{array}\right]_{(30 \times 1)}=\left[\begin{array}{c}
y_{1}^{(1)} \\
y_{1}^{(2)} \\
\vdots \\
y_{1}^{(80)}
\end{array}\right]_{(80 \times 1)}
$$

This can be represented dimensionally as:

$$
[A]_{(80 \times 30)} \times[V]_{(30 \times 1)}=[Z]_{(80 \times 1)}
$$

and expressed in terms of state estimation, it becomes:

$$
[A]_{(80 \times 30)} \times[V]_{(30 \times 1)}+[\eta]_{(80 \times 1)}=[Z]_{(80 \times 1)}
$$

In WLSSE method $A_{e f f e}$ and $Z_{e f f e}$ are calculated as:

$$
\begin{aligned}
{\left[_{\text {effe }}\right]_{(30 \times 30)} } & =\left[A^{T}\right]_{(30 \times 80)} \times\left[R^{-1}\right]_{(80 \times 80)} \times[A]_{(80 \times 30)} \\
& =\left[A^{T}\right]_{(30 \times 80)} \times[A]_{(80 \times 30)} \\
{\left[Z_{\text {effe }}\right]_{(30 \times 1)} } & =\left[A^{T}\right]_{(30 \times 80)} \times\left[R^{-1}\right]_{(80 \times 80)} \times[Z]_{(80 \times 1)} \\
& =\left[A^{T}\right]_{(30 \times 80)} \times[Z]_{(80 \times 1)}
\end{aligned}
$$

In this approach $R^{-1}$ is considered to be the identity matrix:

$$
\left[A_{\text {effe }}\right]_{(30 \times 30)} \times[V]_{(30 \times 1)}=\left[Z_{\text {effe }}\right]_{(30 \times 1)}
$$

Using gauss elimination, vector $[V]$ can be found as:

$$
[V]_{(30 \times 1)}=\left[A_{e f f}\right]_{(30 \times 30)}^{-1} \times\left[Z_{e f f e}\right]_{(30 \times 1)}
$$

It may be noted that the weight vector $[V]_{(30 \times 1)}$ is estimated directly in a non iterative approach using WLSSE technique. The above mentioned process is applied to calculate remaining weights between inputs to hidden neurons. The above process demands the output of hidden layer. The following section discusses the calculation of output of hidden nodes. 


\subsection{Calculation of Hidden Nodes Output}

Since the hidden node values are not known, mean values of input sample would be treated as hidden nodes output.

$$
[Z]_{(80 \times 1)}=\left[\begin{array}{c}
y_{1}^{(1)} \\
y_{1}^{(2)} \\
\vdots \\
y_{1}^{(80)}
\end{array}\right]_{(80 \times 1)}=\left[\begin{array}{c}
\bar{x}^{(1)} \\
\bar{x}^{(2)} \\
\vdots \\
\bar{x}^{(80)}
\end{array}\right]_{(80 \times 1)}
$$

Here $x^{-(i)}=$ Average value of $i^{\text {th }}$ sample $x_{1}^{(i)}, x_{2}^{(i)}, x_{3}^{(i)}$ etc.

And at node 2 output values are given as

$$
\left[\begin{array}{c}
y_{2}^{(1)} \\
y_{2}^{(2)} \\
\vdots \\
y_{2}^{(80)}
\end{array}\right]_{(80 \times 1)}=\left[\begin{array}{c}
r_{t} \cdot \bar{x}^{(1)} \\
r_{t} \cdot \bar{x}^{(2)} \\
: \\
r_{t} \cdot \bar{x}^{(80)}
\end{array}\right]_{(80 \times 1)}
$$

Where, $r_{t}$ is a random number taken between 0.8 to 1.2 , which varies for each $x_{i}^{j}$. A similar approach is adopted for the hidden layer nodes. Each individual $\left[x^{-(i)}\right]$ is multiplied with random value $\left(r_{t}\right)$ in order to obtain the output of hidden node $\left[y^{(i)}\right]$.

$$
\left[y_{i}^{(j)}\right]_{(80 \times 45)}=\left[\begin{array}{cccc}
\bar{x}^{(1)} & r_{t} \cdot \bar{x}^{(1)} & \cdots & r_{t} \cdot \bar{x}^{(1)} \\
\bar{x}^{(2)} & r_{t} \cdot \bar{x}^{(2)} & \cdots & r_{t} \cdot \bar{x}^{(2)} \\
: & : & \cdots & : \\
\bar{x}^{(80)} & r_{t} \cdot \bar{x}^{(80)} & \cdots & r_{t} \cdot \bar{x}^{(80)}
\end{array}\right]_{(80 \times 45)}
$$

Weights between hidden-output nodes in terms of output equations are:

$$
\begin{aligned}
& W_{11} \times y_{1}^{(1)}+W_{21} \times y_{2}^{(1)}+\cdots+W_{451} \times y_{45}^{(1)}=O u t_{1}^{(1)} \\
& W_{11} \times y_{1}^{(2)}+W_{21} \times y_{2}^{(2)}+\cdots+W_{451} \times y_{45}^{(2)}=O u t_{1}^{(2)} \\
& : \\
& : \\
& W_{11} \times y_{1}^{(80)}+W_{21} \times y_{2}^{(80)}+\cdots+W_{451} \times y_{45}^{(80)}=O u t_{1}^{(80)}
\end{aligned}
$$


Matrix representation to the above equations are given below

$$
\left[\begin{array}{cccc}
y_{1}^{(1)} & y_{2}^{(1)} & \cdots & y_{45}^{(1)} \\
y_{1}^{(2)} & y_{2}^{(2)} & \ldots & y_{45}^{(2)} \\
\vdots & \vdots & \cdots & \vdots \\
y_{1}^{(80)} & y_{2}^{(80)} & \cdots & y_{45}^{(80)}
\end{array}\right]_{(80 \times 45)} \times\left[\begin{array}{c}
W_{11} \\
W_{21} \\
: \\
W_{451}
\end{array}\right]_{(45 \times 1)}=\left[\begin{array}{c}
O u t_{1}^{(1)} \\
O u t_{1}^{(2)} \\
: \\
O u t_{1}^{(80)}
\end{array}\right]_{(80 \times 1)}
$$

Out $t_{1}^{(1)}$, Out $_{1}^{(2)} \ldots \ldots$...Out $t_{1}^{(80)}$ are the output of node 1 at output layer for all selected 80 patterns. Here weights are considered as state variables and their representation is given below:

$$
[B]_{(80 \times 45)} \times[W]_{(45 \times 1)}=\left[Z_{o}\right]_{(80 \times 1)}
$$

Above equation in terms of state estimation is

$$
[B]_{(80 \times 45)} \times[W]_{(45 \times 1)}+[\eta]_{(80 \times 1)}=\left[Z_{o}\right]_{(80 \times 1)}
$$

The effective values from WLSSE method is:

$$
\begin{aligned}
{\left[B_{\text {effe }}\right]_{(45 \times 45)} } & =\left[B^{T}\right]_{(45 \times 80)} \times\left[R^{-1}\right]_{(80 \times 80)} \times[B]_{(80 \times 45)} \\
& =\left[B^{T}\right]_{(45 \times 80)} \times[B]_{(80 \times 45)} \\
{\left[Z_{\text {oeffe }}\right]_{(45 \times 1)} } & =\left[B^{T}\right]_{(45 \times 80)} \times\left[R^{-1}\right]_{(80 \times 80)} \times\left[Z_{o}\right]_{(80 \times 1)} \\
& =\left[B^{T}\right]_{(45 \times 80)} \times\left[Z_{o}\right]_{(80 \times 1)}
\end{aligned}
$$

From Equation (16) we have

$$
\begin{aligned}
{\left[B_{\text {eff }}\right]_{(45 \times 45)} \times[W]_{(45 \times 1)} } & =\left[Z_{\text {oeffe }}\right]_{(45 \times 1)} \\
{[W]_{(45 \times 1)} } & =\left[B_{\text {eff }}\right]_{(45 \times 45)}^{-1} \times\left[Z_{\text {oeffe }}\right]_{(45 \times 1)}
\end{aligned}
$$

The weight matrix between hidden and output is obtained from (21). The network is now fully trained with input wind samples and ready for testing on wind speed forecasting. For example if input node is taken as $\left[X_{i}\right]_{(1 \times N)}$ then hidden and output layer are given by

$$
\begin{gathered}
{[Y]_{(1 \times N H)}=\left[X_{i}\right]_{(1 \times N)} \times[V]_{(N \times N H)}} \\
{[\text { Out }]_{(1 \times N o)}=[Y]_{(1 \times N H)} \times[W]_{(N H \times N o)}}
\end{gathered}
$$

Finally the forecasted output is obtained from Equation (23). 


\subsection{Detailed SENN Algorithm steps}

1. Read the Neural Network data

$$
\begin{aligned}
& N \rightarrow \text { No. of Input layer nodes } \\
& N H \rightarrow \text { No. of Hidden layer nodes } \\
& N O \rightarrow \text { No. of Output layer nodes } \\
& N T S \rightarrow \text { No. of Testing Samples } \\
& N S \rightarrow \text { No. of Training Samples }
\end{aligned}
$$

2. Store all the training samples of input in the matrix form as $[A]_{(80 \times 30)}$.

Similarly store the training samples of output in matrix $[T]_{(80 \times 1)}$.

$$
\begin{aligned}
& {[A]_{(80 \times 30)}=\left[\begin{array}{ccccc}
X_{1}^{(1)} & X_{2}^{(1)} & X_{3}^{(1)} & \ldots \ldots & X_{30}^{(1)} \\
X_{1}^{(2)} & X_{2}^{(2)} & X_{3}^{(2)} & \ldots \ldots & X_{30}^{(2)} \\
X_{1}^{(3)} & X_{2}^{(3)} & X_{3}^{(3)} & \ldots \ldots & X_{30}^{(3)} \\
\vdots & \vdots & \vdots & \cdots \cdots & \vdots \\
X_{1}^{(80)} & X_{2}^{(80)} & X_{3}^{(80)} & \cdots \cdots & X_{30}^{(80)}
\end{array}\right]_{(80 \times 30)}} \\
& {[T]_{(80 \times 1)}=\left[\begin{array}{c}
O u t_{1}^{(1)} \\
O u t_{1}^{(2)} \\
O u t_{1}^{(3)} \\
: \\
O u t_{1}^{(N S)}
\end{array}\right]_{(80 \times 1)}}
\end{aligned}
$$

3. Normalize each sample of the input and output.

4. Evaluate the transpose matrix $[A]_{(80 \times 30)}^{T}$.

5. From WLSSE approach calculates $\left[A_{e f f}\right]_{(30 \times 30)}$ using Equation (13).

6. Evaluate output of each node at hidden layer using following relation

$$
\left[y_{i}^{(j)}\right]_{(80 \times 45)}=\left[\begin{array}{cccc}
\bar{x}^{(1)} & r_{t} \cdot \bar{x}^{(1)} & \cdots & r_{t} \cdot \bar{x}^{(1)} \\
\bar{x}^{(2)} & r_{t} \cdot \bar{x}^{(2)} & \cdots & r_{t} \cdot \bar{x}^{(2)} \\
: & : & \cdots & \vdots \\
\bar{x}^{(80)} & r_{t} \cdot \bar{x}^{(80)} & \cdots & r_{t} \cdot \bar{x}^{(80)}
\end{array}\right]_{(80 \times 45)}
$$

7. Calculate $Z_{\text {eff }}$ using Equation (12)

$$
\left[Z_{e f f}\right]_{(30 \times 1)}=\left[A^{T}\right]_{(30 \times 80)} \times[Z]_{(80 \times 1)}
$$


8. Using Gauss elimination technique calculate the weight matrix $[V]_{(30 \times 1)}$ between input to hidden node using Equation (14) and repeat this procedure for remaining hidden nodes.

9. Compute the weights between hidden and output layer, by representing hidden layer output and target output in matrix form as shown below:

$$
\begin{aligned}
& {[B]_{(80 \times 45)}=[y]_{(80 \times 45)}} \\
& {\left[Z_{o}\right]_{(80 \times 1)}=[T]_{(80 \times 1)}}
\end{aligned}
$$

10. Compute the $\left[B_{\text {eff }}\right]$ and $\left[Z_{o e f f}\right]$ matrices using Equations (18) and (19).

11. Using gauss elimination technique calculate the weights $[W]_{(45 \times 1)}$ between hidden and output node using Equation (21).

12. Load the test samples from test input file. Input sample $=\left[X_{i}\right]_{(1 \times 30)}$; Target Output $=[T(O)]_{(1 \times 1)}$

13. Normalize the input samples and actual output.

14. Calculate output of hidden layer by multiplying with weights $[V]_{(30 \times 45)}$ using following relation

$$
[y]_{(1 \times 45)}=\left[x_{i}\right]_{(1 \times 30)} \times[V]_{(30 \times 45)}
$$

15. Output results of output layer is calculated by multiplying with weights $[W]_{(45 \times 1)}$ using following relation

$$
[\text { Out }]_{(1 \times 1)}=[y]_{(1 \times 45)} \times[W]_{(45 \times 1)}
$$

16. De-normalize the forecasted output and repeat this procedure for next 24 hours.

\section{Results and Discussion}

In this paper, in order to perform the forecasting analysis, practical wind speed data has been taken from National Renewable Energy Laboratory (NREL) website [24]. In this section BPNN, SENN based wind speed forecasting results have been compared and discussed. For both BPNN and SENN same network architecture has been used for forecasting application. For BPNN network, learning rate $=0.8$, momentum factor $=0.2$, epsilon (tolerance) $=0.0001$ and maximum numbers of iterations are set as 400. Number of training patterns is 80 and testing patterns are 24 in both cases. In this problem BPNN has converged in 340 iterations [25]. Using single statistical parameter analyzing forecasting efficiency is not much effective, so in this section set of statistical parameters are considered to evaluate forecasting accuracy. 
Forecasting accuracy is evaluated by following statistical parameters such as:

- Absolute Percentage Error $A P E=\left|\frac{A W-F W}{A W}\right|$

- Mean Absolute Percentage Error $M A P E=\frac{1}{n} \sum_{i=1}^{n}\left|\frac{A W-F W}{A W}\right| * 100$

- Mean Absolute Error $M A E=\frac{1}{n} \sum_{i=1}^{n}|A W-F W|$

- Root Mean Square Error $R M S E=\sqrt{\sum_{i=1}^{n} \frac{(A W-F W)^{2}}{n}}$

- Correlation Coefficient $R=\frac{R_{A F}}{\operatorname{Std}(A) * \operatorname{Std}(F)}$

In the above equations the following symbols are used, namely $A W=$ Actual wind speed, $F W=$ Forecasted wind speed, $n=$ Number of wind samples, $R_{A F}=$ Covariance between Actual and Forecasted wind speed, Std $(A)=$ Standard deviation of Actual wind speed, $\operatorname{Std}(F)=$ Standard deviation of Forecasted wind speed.

Table 1 shows comparison of actual and predicted wind speed using BPNN and SENN where MAPE is obtained as 13.221 and 8.173 respectively and individual APE also been calculated for each (total 24) wind sample. Figure 3 is the graphical representation of Table 1 . Table 2 reports the comparison of statistical measures such as MAPE, MAE, RMSE, Correlation coefficient (R) and the execution time.

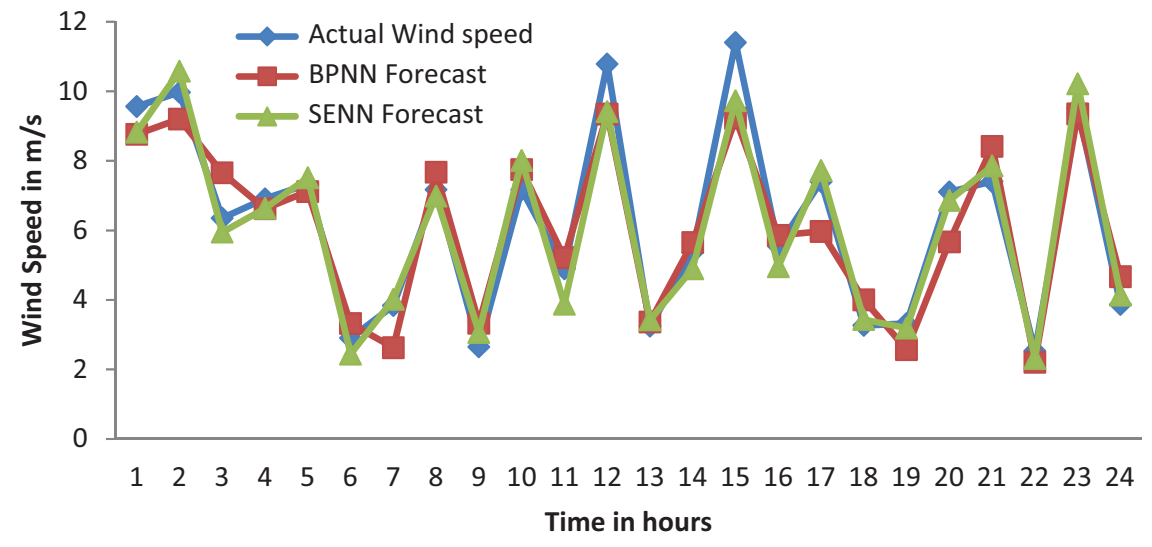

Figure 3 Actual and forecasted wind speed for BPNN and SENN. 
Table 1 Comparison of actual and forecasted wind speed using BPNN, SENN

\begin{tabular}{|c|c|c|c|c|c|}
\hline S.No. & $\begin{array}{l}\text { Actual Wind } \\
\text { Speed }(\mathrm{m} / \mathrm{s})\end{array}$ & $\begin{array}{c}\text { Forecasted Speed } \\
\text { BPNN }(\mathrm{m} / \mathrm{s})\end{array}$ & $\begin{array}{c}\text { Forecasted Speed } \\
\text { SENN }(\mathrm{m} / \mathrm{s})\end{array}$ & $\begin{array}{c}\text { APE } \\
\text { BPNN }\end{array}$ & $\begin{array}{l}\text { APE } \\
\text { SENN }\end{array}$ \\
\hline 1 & 9.563 & 8.762 & 8.824 & 8.376 & 7.727 \\
\hline 2 & 9.972 & 9.203 & 10.578 & 7.712 & 6.077 \\
\hline 3 & 6.352 & 7.655 & 5.948 & 20.513 & 6.360 \\
\hline 4 & 6.909 & 6.624 & 6.634 & 4.125 & 3.980 \\
\hline 5 & 7.328 & 7.117 & 7.516 & 2.879 & 2.565 \\
\hline 6 & 2.905 & 3.321 & 2.443 & 14.320 & 15.903 \\
\hline 7 & 3.838 & 2.625 & 4.015 & 31.605 & 4.611 \\
\hline 8 & 7.175 & 7.671 & 6.985 & 6.912 & 2.648 \\
\hline 9 & 2.65 & 3.327 & 3.065 & 25.547 & 15.660 \\
\hline 10 & 7.175 & 7.7471 & 8.011 & 7.973 & 11.651 \\
\hline 11 & 4.894 & 5.216 & 3.875 & 6.579 & 20.821 \\
\hline 12 & 10.788 & 9.346 & 9.415 & 13.366 & 12.727 \\
\hline 13 & 3.246 & 3.365 & 3.424 & 3.666 & 5.483 \\
\hline 14 & 5.367 & 5.656 & 4.895 & 5.384 & 8.794 \\
\hline 15 & 11.406 & 9.224 & 9.725 & 19.130 & 14.737 \\
\hline 16 & 5.547 & 5.859 & 4.955 & 5.624 & 10.672 \\
\hline 17 & 7.403 & 5.971 & 7.724 & 19.343 & 4.336 \\
\hline 18 & 3.267 & 4.001 & 3.435 & 22.467 & 5.142 \\
\hline 19 & 3.32 & 2.57 & 3.187 & 22.590 & 4.006 \\
\hline 20 & 7.107 & 5.668 & 6.862 & 20.247 & 3.447 \\
\hline 21 & 7.406 & 8.417 & 7.855 & 13.651 & 6.062 \\
\hline 22 & 2.531 & 2.208 & 2.314 & 12.761 & 8.573 \\
\hline 23 & 9.525 & 9.349 & 10.218 & 1.847 & 7.275 \\
\hline 24 & 3.868 & 4.668 & 4.135 & 20.682 & 6.902 \\
\hline MAPE & & & & 13.221 & 8.173 \\
\hline
\end{tabular}

Table 2 Comparison of statistical parameters using BPNN and SENN

\begin{tabular}{lcc}
\hline Statistical Measures & BPNN Forecast & SENN Forecast \\
\hline MAPE & 13.221 & 8.173 \\
\hline MAE & 0.753 & 0.504 \\
\hline RMSE & 0.910 & 0.635 \\
\hline R & 0.904 & 0.933 \\
\hline Execution Time & $68 \mathrm{sec}$. & $29 \mathrm{sec}$. \\
\hline
\end{tabular}

Comparison of all statistical measures prove that, SENN based wind speed forecasting method is showing better performance when compared to BPNN based wind speed forecasting method. In particular, statistical data prove that SENN based forecasting are more accurate and they have more correlation to the original wind samples. Indeed, Figure 3 shows that SENN based forecasting method results are coinciding with actual wind speed values 
more closely where as BPNN results are deviating to the original wind speed values. In BPNN, weights are estimated randomly and error is back propagated to adjust the weights. In SENN technique weights are estimated accurately based on weighted least square approach. SENN is a non iterative technique and it will take less time of training for same error when compared with BPNN method. Run time for SENN is low when compared to BPNN because of non iterative nature and deterministic weight approach of SENN, which is reduced to $57.3 \%$ as compared to that of BPNN.

\section{Conclusion}

In this paper day ahead (24 hours) wind speed forecasting has been carried out using BPNN and SENN based methods. Both these methods have been tested with practical data from NREL website. Statistical measures illustrate that SENN method has least error and an improved correlation factor so that forecasting results are more accurate and coinciding with actual wind values. In BPNN weights are initialized randomly and error is back propagated to adjust the weights. In SENN technique weights are estimated accurately based on the weighted least square approach because of which this method is more accurate. BPNN is a traditional forecasting method where the weights are updated based on error and back propagated to reach desired value and it suffers with local minima problem. SENN is a complete mathematical approach and weights are estimated between layers accurately using weighted least square technique, therefore error is reduced. Execution time (training as well as testing) for SENN is comparatively low as that of BPNN since SENN method is a non-iterative technique and it can be used for online studies like contingency analysis.

\section{References}

[1] Haque, A. U., Nehrir, M. H., and Mandal, P. (2014). A hybrid intelligent model for deterministic and quantile regression approach for probabilistic wind power forecasting. IEEE Trans. Power Syst., 29(4), 1663-1672.

[2] Khosravi, A., Nahavandi, S., and Creighton, D. (2013). Prediction intervals for short-term wind farm power generation forecasts. IEEE Trans. Sustain. Energy, 4(3), 602-610.

[3] Bhaskar, K., and Singh, S. N. (2012). AWNN-assisted wind power forecasting using feed-forward neural network. IEEE Trans. Sustain. Energy, 3(2), 306-315. 
[4] Chandra, D. R., Kumari, M. S., Sydulu, M., Grimaccia, F., and Mussetta, M. (2014). Adaptive wavelet neural network based wind speed forecasting studies. J. Electr. Eng. Technol.,. 9(6), 1812-1821.

[5] R Ramesh, B., and Arulmozhivarman, P. (2013). Improving forecast accuracy of wind speed using wavelet transform and neural networks. J. Electr. Eng. Technol., 8(3), 559-564.

[6] Yao, C., Gao, X., and Yu, Y. (2013). Wind speed forecasting by wavelet neural networks: a comparative study. Math. Probl. Eng., 2013.

[7] Pindoriya, N. M., Singh, S. N., and Singh, S. K. (2008). An adaptive wavelet neural network-based energy price forecasting in electricity markets. IEEE Trans. Power Syst., 23(3), 1423-1432.

[8] Sideratos, G., and Hatziargyriou, N. D. (2012). Wind power forecasting focused on extreme power system events. IEEE Trans. Sustain. Energy, 3(3), 445-454.

[9] Chandra, D. R., Kumari, M. S., and Sydulu, M. (2013). A detailed literature review on wind forecasting. In International Conference on Power, Energy and Control (ICPEC), (pp. 630-634). IEEE.

[10] Wu, Y. K., and Hong, J. S. (2007). A literature review of wind forecasting technology in the world. In IEEE Lausanne Power Tech, (pp. 504-509). IEEE.

[11] El-Fouly, T. H., El-Saadany, E. F., and Salama, M. M. (2008). One day ahead prediction of wind speed and direction. IEEE Trans. Energy Convers., 23(1), 191-201.

[12] Ernst, B., Oakleaf, B., Ahlstrom, M. L., Lange, M., Moehrlen, C., Lange, B., Focken, U., and Rohrig, K. (2007). "Predicting the wind," IEEE Power Energy Mag., 5(6), 78-89.

[13] Kusiak, A., Zheng, H., and Song, Z. (2009). Short-term prediction of wind farm power: A data mining approach. IEEE Trans. Energy Convers., 24(1), 125-136.

[14] Barbounis, T. G., Theocharis, J. B., Alexiadis, M. C., and Dokopoulos, P. S. (2006). Long-term wind speed and power forecasting using local recurrent neural network models. IEEE Trans. Energy Convers., 21(1), 273-284.

[15] Ouammi, A., Dagdougui, H., and Sacile, R. (2012). Short term forecast of wind power by an artificial neural network approach. In IEEE International Systems Conference (SysCon), (pp. 1-5). IEEE.

[16] Monfared, M., Rastegar, H., and Kojabadi, H. M. (2009). A new strategy for wind speed forecasting using artificial intelligent methods. Renewable Energy, 34(3), 845-848. 
[17] Shi, J., Guo, J., and Zheng, S. (2012). Evaluation of hybrid forecasting approaches for wind speed and power generation time series. Renew. Sustain. Energy Rev., 16(5), 3471-3480.

[18] Wan, Y., and Zhang, H. (2012). Application of artificial intelligence to wind forecasting: An enhanced combined approach. In 8th International Conference on Natural Computation (ICNC), (pp. 385-388). IEEE.

[19] Çevik, H. H., and Çunkaş, M. (2015). Short-term load forecasting using fuzzy logic and ANFIS. Neural Computing and Applications, 26(6), 1355-1367.

[20] Wu, Y. K., Lin, C. L., Ke, B. R., Huang, Y. K., and Hsu, K. W. (2010). Development of an industrial forecasting tool in wind power. In 5th IEEE Conference on Industrial Electronics and Applications (ICIEA), (pp. 614-619). IEEE.

[21] Zhang, Y., and Chan, K. W. (2008). The impact of wind forecasting in power system reliability. In International Conference on Electric Utility Deregulation and Restructuring and Power Technologies (DRPT 2008). (pp. 2781-2785). IEEE.

[22] Morina, M., Grimaccia, F., Leva, S., and Mussetta, M. (2016). Hybrid weather-based ANN for forecasting the production of a real wind power plant. In International Joint Conference on Neural Networks (IJCNN), (pp. 4999-5005). IEEE.

[23] Prakash, K., and Sydulu, M. (2009). Non iterative-state estimation based neural network for short term load forecasting of distribution systems. In Power \& Energy Society General Meeting,. PES'09. (pp. 1-8). IEEE.

[24] Available at: http://www.nrel.gov/wind/integrationdatasets/eastern/data. html

[25] Rakesh Chandra, D. (2017). "Wind integration studies in Power systems: Forecasting, Stability and load managemnt issues" LAP Lambert publishers, Germany. 


\section{Biographies}

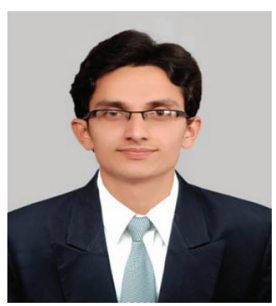

D. Rakesh Chandra received the B.Tech degree from VNR Vignana Jyothi Engineering College, Hyderabad, India, in 2008, and M. Tech and Ph.D degrees from National Institute of Technology, Warangal, India, in 2010 and 2016 respectively. He has been working as assistant professor in the department of EEE at Kakatiya Institute of Technology \& Science, Warangal since 2016. He received prestigious POSOCO award in the year 2017. His research interests are in the area of integration of Renewable Energy Sources, Energy storage and smart grids.

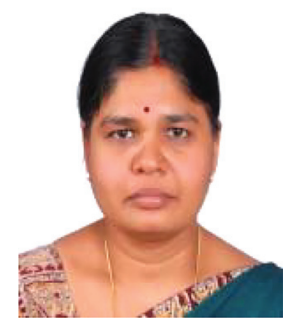

M. Sailaja Kumari obtained her B.E and M.E degrees from University College of engineering, Osmania University, Hyderabad, Andhra Pradesh, India in 1993, 1995 and Ph.D in 2008 from National Institute of Technology, Warangal. She joined Department of Electrical Engineering, National Institute of Technology Warangal, India in 1997 as Lecturer. Currently she is Professor in the Dept. of Electrical Engineering, NIT Warangal. Her research interests are in the area of Power system Deregulation, Transmission pricing, renewable energy sources and Application of neural networks and genetic algorithms in power systems. 


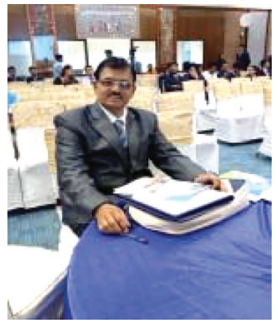

M. Sydulu obtained his B.Tech (Electrical Engineering, 1978), M.Tech (Power Systems, 1980), Ph.D (Electrical Engineering - Power Systems, 1993), all degrees from Regional Engineering College Warangal, Andhra Pradesh, India. His areas of interest include Real Time power system operation and control, ANN, fuzzy logic and Genetic Algorithm applications in Power Systems, Distribution system studies, Economic operation, Reactive power planning and management. Presently he is Professor of Electrical Engineering, at National Institute of Technology, Warangal (formerly RECW).

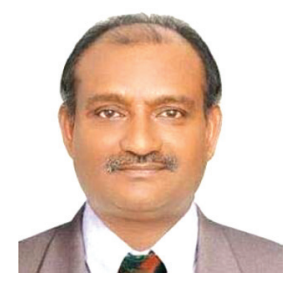

V. Ramaiah working as a Professor \& Head of Electrical and Electronics Engineering Department in KITS-Warangal. He is pursuing Ph.D in the field of Performance Analysis of Electric Distribution utilities at VTU, Chennai and obtained his M.E degree from BITS-Pilani. He has around 25 years of experience in teaching \& Research in Electrical and Electronics Engineering at KITS Warangal and also worked as Scientist (Fellow) at CEERI, Pilani(Raj). 
